\title{
Management of Epileptic Patients in Dentistry
}

\author{
Yaltırık Mehmet $^{1}$, Özer Senem ${ }^{1}$, Tonguç Sülün ${ }^{2}$, Kocaelli Hümeyra ${ }^{1}$ \\ ${ }^{1}$ Department of Oral Surgery, Faculty of Dentistry, Istanbul University, Istanbul, Turkey \\ ${ }^{2}$ Department of Prosthodontics, Faculty of Dentistry, Istanbul University, Istanbul, Turkey \\ Email: \{myaltrk, tongucsulun, humeyrakocaelli\}@yahoo.com, senemozr@hotmail.com
}

Received June 9, 2011; revised September 23, 2011; accepted October 13, 2011

\begin{abstract}
Epilepsy has direct negative effects on sufferers' general dental condition and oral health, both of which are further affected by inadequate oral hygiene; poor oral hygiene itself is often also caused by epilepsy-related poor health. Consequently, tooth loss, caries and periodontal disease occur increasingly often in epilepsy sufferers and they need more dental treatment. However, in fact the epileptic patients can receive fewer and simpler treatment modalities. The aim of this study was to review and synthesize recent studies on dental treatment in epilepsy patients and to mention potential triggers for seizures in dental practice.
\end{abstract}

Keywords: Epilepsy; Dental; Seizure; Prosthodontic Treatment

\section{Introduction}

The human brain consists of millions of neurons, their extensions, and the supportive tissues found between those neurons. All brain cells have the ability to produce electrical currents and conduct them to other cells. It is by transmitting such electrical signals that the brain functions. In other words, it is the conduction of these electrical currents that enables us to act, to speak and to feel $[1,2]$.

Seizures can be defined as the discontinuity of normal brain functions due to sudden electrical discharges which may be either excessive or inadequate; these result in episodic convulsions (such as involuntary motion), disturbances in perception, or alterations in consciousness. The outcome of such excessive discharge during electrical conduction is called seizure [2-4].

Epilepsy is a disease that involves seizures which are characterized by an alteration of perception, behavior and mental activities, as well as by involuntary muscle contractions, temporary loss of consciousness and chronic changes in neurological functions that result from abnormal electrical activity in the brain [3,5,6]. Epileptic seizures are reversible and recur frequently [2].

For centuries epilepsy was thought to be a disease related to the supernatural. Although Hippocrates claimed that epilepsy is a naturally occurring disease, the misbelieve that the cause of epilepsy was supernatural was common until the neuropathologic origin of epilepsy was reported in the 19th century [7]. In the early part of that century, John Hughlings Jackson defined Jocksonian seizures; in the middle of the $19^{\text {th }}$ century, Robert Bently
Todd defined the paralyses that may develop after longterm seizures [2]. Today, the diagnosis of an epileptic patient required at least three seizure episodes [1].

The aim of this study was to review and synthesize recent studies on dental treatment in epilepsy patients and outline the special concerns that dentists should take into account when providing care to these patients.

We searched the dental literature with Medline/Pubmed with an emphasis on peer-reviewed journals and Science Citation Index Expanded. Key words used were epilepsy, dental, seizure and prosthodontic treatment. We also scrutinized common textbooks on removable and fixed proshodontics. For additional information a hand search from relevant data were searched, too.

\section{Epidemiology and Prevalence}

Epilepsy is a disease that is frequently encountered by oral and maxillofacial surgery practices [2]. It is thought to affect millions of people worldwide, and has a prevalence of $0.5 \%-0.9 \%$ in the general population $[1,2,8]$. Chapman et al. have reported that, epileptic seizures are the second most common medical incident in dental surgeries. They have stated that statistically every dentist notice in his/her professional life 1.5 times generalized tonic-clonic seizures by the patients [9].

It has been reported that the disease occurs independent of race, age and gender [2,10]. However, epilepsy has been occur more frequently in men than in women $[2,5$, 10,11].

Epilepsy has been observed most frequently in children under 1 year of age and in people over the age of 75 
$[2,10,11]$. It has also been reported that those cases of epilepsy that develop in childhood are genetic in origin, while those that appear in adulthood are related to cerebrovascular diseases [2,10]. Up to $50 \%$ of children displaying mental retardation have been diagnosed as suffering from epilepsy $[4,10,11]$.

\subsection{Etiology}

In $70 \%$ of epilepsy cases, the specific etiology is not known for certain. These cases are defined as idiopathic or primary epilepsy. When the etiology of seizures is known, the condition is known as secondary or acquired epilepsy [2,10,11].

Secondary epilepsies are the result of metabolic, genetic, structural or functional anomalies [2]. Examples of metabolic disorders related with epilepsy are electrolyte imbalance, acidosis, hyperglycemia, hypoglycemia, hypoxia, dehydration, water intoxication [2,10,11]. Examples of systemic disorders that cause seizures are sepsis, systemic lupus erythematosus, hypertension and diabetes [2]. Other causes of epilepsy are cerebral vascular malformations, brain tumors and hamartomas, hypocsicischemic lesions, head traumas, birth traumas, infections affecting babies during pregnancy, inflammatory diseases and infectious diseases [2]. Seizures can also be associated with West Syndrome, Lennox-Gastaut Syndrome and Landau Kleffner Syndrome [2,12,13]. There are also some case reports of reflex epileptic seizures caused by prolonged brushing of teeth [14-17].

The etiology of secondary epilepsy can be determined only in one-third to one-half of the cases diagnosed when patients are adult. Cerebrovascular diseases are the most frequently encountered causes of epilepsy (40\% of cases). The second most frequently encountered cause of epilepsy is brain tumors [2,10].

Epilepsy genes are related to mutations, which cause abnormal brain ontogenesis, neurodegeneration and abnormal functioning. There are three types of epilepsy which show autosomal transmission [2]:

- Benign familial neonatal seizures;

- Generalized epilepsy with inflammatory seizures;

- Partial epilepsy syndrome/nocturnal frontal lob epilepsy.

\subsection{Classification}

Epilepsy is classified according to seizure type: generalized type, partial type, and status epilepticus, [2,5,18,19] as described below.

\subsubsection{Generalized Type}

In the generalized type of epilepsy, each hemisphere of the brain is affected synchronously. There is usually no indication (aura) before the seizure and the patient is un- conscious during the seizure [2]. The generalized type is classified into three groups: generalized tonic-clonic seizures (grand mal), atonic seizures, and absence seizures (petit mal) [2,5,12].

\subsubsection{Partial Type}

The partial type of epilepsy emerges only when a particular area of the cerebral cortex is affected; it can be diagnosed on the basis of electroencephalogram (EEG) results and clinical symptoms. This type is classified into three groups: simple partial seizures, complex partial seizures and secondarily generalized seizures $[2,5,18]$.

\subsubsection{Status Epilepticus}

"Status epilepticus" is a term that used to describe any continuous seizure. It arises frequently after tonic-clonic seizure. It can be fatal if not treated $[2,3,5,18,20]$.

Factors Provoking Seizures

The following factors may all provoke seizures:

- Incorrect use of medication;

- Sleep deprivation;

- Drug abuse;

- Excessive use of alcohol;

- Use of medication that reduces the effectiveness of antiepileptic medicines;

- Excessive use of caffeine;

- Hormonal changes;

- Low blood sugar level (hypoglycemia);

- Deficiency of vitamin B6;

- Electrolyte imbalance in the blood;

- Congenital diseases: neurofibromatosis, tuberous sclerosis, phenylketonuria [2,5].

\section{Oral and Maxillofacial Treatment of Epileptic Patients}

When treating epileptic patients, the major difficulty a dentist faces is the high risk of seizures occurring. In order to prevent such seizures, three fundamental principles should guide the dentist during his or her interaction with the patient in a clinical environment: 1) knowledge of the patient's previous seizure episodes and medication, 2) knowledge of the conditions that provoke epileptic seizures, in order to avoid such conditions, and 3) dentist should be able to recognize the early signs of a seizure, take precautions before it occurs, and provide the patient with supportive care if it does occur [3-5,19].

Anti-epileptic drugs (AEDs) can also have side effects which have a direct bearing on dental treatment. Phenytoin, for example, has the most widely known side effect, as it causes gingival hyperplasia. It has been reported that this side effect is observed in $50 \%$ of patients taking this medicine for three months [5,6,13,21-27]. Carbamazepine, another AED, causes xerostomia, ulcer, glossitis and sto- 
matitis [5,21,24].

Sodium valproate may reduce the effectiveness of the blood-clotting mechanism $[5,24]$. It has been recommended that, if a major operation is planned, the patient should consult his/her doctor and precautions should be taken; however, this is not essential in the case of minor operations such as tooth removal [5,24]. In addition, Phenobarbital-induced multiform erythema causes bullous and eruption formation and primidone has been reported to cause megaloblastic anemia [2,24].

It has been reported that most of the medicines prescribed by dentists react with AEDs and reduce the effects of those AEDs. It is stated that non-steroidal antiinflammatory drugs and some of antifungals such are metronidazole, fluconazole and miconazole affects the metabolism of carbamazepine sodium valproate and phenytoin negatively. Therefore, it has been stressed that these drug combinations need to be avoided $[2,5,18]$.

\subsection{Factors to Be Taken into Consideration While Administering Treatment}

Before starting treatment, a detailed patient anamnesis should be obtained $[3,5,18,28]$. This should cover the following:

- The frequency of seizures;

- The date of the patient's last seizure;

- The consciousness and respiratory state of the patient during seizures;

- The physical condition of the patient after a seizure;

- Whether there is any aura before seizures;

- Whether experiencing an aura always leads to a seizure;

- The factors provoking seizures;

- The existence of status epilepticus.

As stress is one of the most important factors that provoke seizures, stress-causing factors should be eliminated before starting the treatment. The patient's appointment should be in the early hours of the day, treatment sessions should be kept short, and sudden stimulants such as shimmering bright lights and extreme noise should be avoided $[5,10,28]$. Furthermore, even though they may be taking medication, if patients are having more than one seizure per month, their treatment must be postponed unless it is urgent $[5,10,18,28]$.

\subsubsection{Local Anesthesia}

With regard to the safety of administering local anesthesia to epileptic patients, it has been reported that the issue is still inconclusive and not certain [29]. Other authors have also stated that there is no evidence available concerning the convulsive effects of low-dosage anesthetic administration during dental treatment [5].

Kennedy et al. [12] stated that, in dental practices, lo- cal anesthetics administrated in therapeutic dosages do not interact with standard antiepileptic drugs. In the case of a critical overdose of local anesthetic, however, clinical conditions such as generalized tonic-clonic convulsions may be observed $[12,29]$. Although it has been reported that local anesthetic administration during dental treatment is safe, [29] it is also proposed that adrenalin in local anesthetics has a potential affect on epileptic seizures by intravenous injection [20].

\subsubsection{General Anesthesia}

General anesthesia has been said to be the right choice if epileptic seizures are difficult to bring under control [5]. Furthermore, if a patient is mentally retarded, then general anesthesia should be considered necessary in view of the fact that a seizure may be triggered by stress due to difficulties in communication $[5,28,29]$.

It has been reported that no complications have been encountered under general anesthesia during the treatment of patients who use anti-convulsive medications regularly [5]. However, local anesthesia should be preferred to general anesthesia as far as possible during the treatment of epileptic patients. This is because the brain may suffer from temporary anoxia during general anesthesia, which may initiate epileptic seizures [29].

\subsubsection{Conscious Sedation}

During dental treatment, it has been suggested that seizure development can be controlled by sedation through nitrous oxide inhalation or intravenous benzodiazepine sedation $[5,12]$. However, sometimes a seizure may develop during dental treatment despite intravenous sedation; in such a case treatment must be postponed [5,12].

\subsubsection{Trauma}

During seizures, injuries such as soft tissue damage, tongue injuries, fractures within the maxillofacial region, temporomandibular joint subluxations, tooth fractures, subluxation or avulsion frequently occur $[5,30]$. A broken tooth should be treated following normal procedures but re-implantation of an avulsed tooth should be avoided [5]. If an avulsed tooth cannot be found, then a radiograph is recommended, given the risk that the patient may have swallowed or inhaled the tooth [5,31].

Phenytoin and phenobarbital both increase epileptic patients' risk of fractures, as these drugs accelerate the excretion and metabolism of vitamin D. For these patients, therefore, a combination of prophylactic anti-epilepsy drugs, in tandem with vitamin $\mathrm{D}$ and calcium supplements, is recommended [32]. When treating a patient who has little or no control over their seizures, and when dealing with maxillofacial fractures, it is crucial to make sure that the patient's airway is not blocked. When operating on maxillomandibular fractures, open reduction and 
fixation should be preferred and bimaxillary fixation should be avoided [2,32,33].

Added to these, it also reported that biting the finger of the dentist by the patient during seizure is common. This type of trauma have a risk of infection for the dentist, that is not directly belonging to the epilepsy itselfes [17].

\subsubsection{Protective Treatment}

Factors such as toothache and oral infection, which cause pain and make the patient uncomfortable, may provoke epileptic seizures. It is possible to treat and eliminate these factors during periodic dentist appointments, before complications arise [3,34].

The degree of gingival hyperplasia caused by phenytoin should be controlled by preventing the formation of plaque $[3,5,22,23]$. However, plaque removal would be ineffective unless hyper-plastic tissue is properly removed during gingivectomy. If hyperplasies recur, the patient should change his medication in consultation with his doctor $[5,22,23]$.

Research indicates that epileptic patients have severely inadequate mouth hygiene, oral health and dental condition, as compared with nonepileptic patients. This is explained by the fact that these patients receive insufficient dental care because they spend only a short time in the dentist's chair due to the risk of seizure. Furthermore, their dental condition is worsened by injuries and damage caused to both hard and soft tissues in the maxillofacial region during seizures. Therefore, protective methods such as the use of chlorohexidine and fluoride, education regarding oral hygiene, regular dental check-ups, and educating children to avoid sugary foods and drinks are crucially important $[1,3,5,21,28,34]$.

\subsubsection{Restorative Treatment}

Treatment should be determined according to the type and intensity of patients' seizures. Metal temporary crowns or implant-supported-bridges are more practical than amalgam or porcelain restorations because of the risk of damage during seizures [5,21]. It has been reported that recently developed composite materials are beneficial for incisor teeth restoration [5]. A rubber dam must be used in light of the risk of seizure during restorative treatment $[4,5]$.

\subsubsection{Prosthetic Treatment}

The results of the study undertaken by Karolyhazy et al. [21] have shown that epileptic patients face a higher risk of tooth loss and lose their teeth earlier than the general population; they also receive less prosthetic rehabilitation. Another finding of those authors was that, for fixed prosthesis, a nickel-chrome composite material is used more frequently than a metal-ceramic composite material. The above observations were suggested to be dependent on the patient's socioeconomic status and to arise from inadequate protective, restorative, and prosthodontic care [28].

It is important that the type of denture provided to an epileptic patient is based on the type of seizure they experience. However, in relation to denture provision, special treatment is not required for patients who have not suffered from a seizure for a long period of time (whether or not they are on medication) or for patients who do not experience involuntary masticatory strokes during seizure [28]. In fact, $70 \%$ of epileptic patients fall into this category of not requiring special treatment with regard to denture provision [28].

By contrast, patients who suffer from tonic-clonic seizures with involuntary muscle contractions that include the masticatory system require special treatment. This is because there is a risk of the inhalation of and damage to the prosthesis if it falls out of its normal position during seizure [28,35]. Sanders et al. [3] advised that a fixed prosthesis, instead of a removable prosthesis, should be used for such patients. In cases in which medical conditions dictate the use of a removable prosthesis, dentures must be supported on a metallic base. If the dental interstice is broad, telescopic supporting dentures with a metallic base would also be beneficial [28]. It is also proposed that a good denture and oral hygiene and a good fit of the dentures decrease the risk of phenytoin induced gingival hyperplasia [35].

\subsubsection{Orthodontic Treatment}

Orthodontic treatment can be conducted easily in epileptic patients. A fixed appliance should be preferred over a removable appliance. Otherwise, a removable appliance must have maximum retention [3,5]. An occlusal splint must also be retentive when used to treat epileptic patients who have temporomandibular disorders [28].

\subsection{Actions to Be Taken If a Patient Has an Epileptic Seizure during Dental Treatment}

The appointment should be given to the patient in early hours of the day, treatment sessions should be kept short and sudden stimulants like shimmering bright lights and extreme noise should be avoided [5].

- Treatment should be stopped and dental tampons, prostheses and instruments should be removed [3,28].

- The patient should be helped into the supine position.

- It is not necessary to restrain the patient or move him/her to the floor; neither is it necessary to place an object in the patient's mouth (e.g. to stop them swallowing their tongue). Preventing injury is the primary, most important goal of assistance [3].

- Any tight clothing the patient is wearing should be loosened [3]. 
- If the patient has an aura, thick gauze tampons should be placed in the patient's mouth in order to prevent any kind of injury or damage to the tooth $[5,18]$.

- Some patients fall into a deep sleep after seizure. In such cases the patient must be monitored closely [5, 12].

- If the seizure lasts more than 3 minutes and recurs, drug administration is required.

- The patient should be monitored to make sure his/her airway does not become obstructed. However, if the seizure lasts longer and continues in spite of medication, the patient should be sent to hospital $[1,3,18]$.

- After the patient regains consciousness, he/she should be sent home to rest. Treatment should be postponed until the patient feels well again $[1,3,5,18,28]$.

\section{Conclusion}

As a result of poor oral hygiene, dental trauma during seizures and the side effects of the medication for the epileptic patients; tooth loss, caries and periodontal disease found more frequently in epilepsy sufferers than in non-sufferers. For that reason, the epileptic patients need more dental treatment then other patients. However, it is a fact that special treatment is not required for patients who have not suffered from a seizure for a long period of time or for patients who do not experience involuntary masticatory strokes during seizure. Consequently, after a detailed anamnesis and examination, most epileptic patients can receive a better dental management than that they can usually have.

\section{REFERENCES}

[1] N. Aydemir, C. Ozkara, R. Canbeyli and A. Tekcan, "Changes in Quality of Life and Self-Perspective Related to Surgery in Patients with Temporal Lobe Epilepsy," Epilepsy \& Behavior, Vol. 5, No. 5, 2004, pp. 735-742. doi:10.1016/j.yebeh.2004.06.022

[2] M. D. Turner and R. S. Glickman, "Epilepsy in the Oral and Maxillofacial Patient: Current Therapy,” Journal of Oral and Maxillofacial Surgery, Vol. 63, No. 7, 2005, pp. 996-1005. doi:10.1016/j.joms.2004.04.038

[3] B. J. Sanders, J. A. Weddell and N. N. Dodge, "Managing Patients Who Have Seizure Disorders: Dental and Medical Issues," Journal of the American Dental Association, Vol. 126, No. 12, 1995, pp. 1641-1647.

[4] H. B. Waldman, M. Swerdloff and S. P. Perlman, "Children with Mental Retardation and Epilepsy: Demographics and General Concerns," ASDC Journal of Dentistry for Children, Vol. 67, No. 231, 2000, pp. 268-274.

[5] J. Fiske and C. Boyle, "Epilepsy and Oral Care," Dental Update, Vol. 29, 2002, pp. 180-187.

[6] E. O. Ogunbodede, B. Adamolekun and A. O. Akintomide, "Oral Health and Dental Treatment Needs in Nigerian Patients with Epilepsy,” Epilepsia, Vol. 39, No. 6,
1998, pp. 590-654. doi:10.1111/j.1528-1157.1998.tb01426.x

[7] F. M. Mai, "Psychiatric Comorbidity in Epilepsy: Basic Mechanisms, Diagnosis, and Treatment. Book Review," Journal of Psychiatry and Neuroscience, Vol. 23, 1998, pp. 314-315.

[8] T. R. Browne and G. L. Holmes, “Epilepsy,” New England Journal of Medicine, Vol. 344, No. 15, 2001, pp. 1145-1151. doi:10.1056/NEJM200104123441507

[9] P. J. Chapman, "Medical Emergencies in Dental Practice and Choice of Emergency Drugs and Equipment: A Survey of Australien Dentists," Australian Dental Journal, Vol. 42, No. 2, 1997, pp. 103-108. doi:10.1111/j.1834-7819.1997.tb00104.X

[10] A. H. Friedlander and J. L. Cummings, “Temporal Lobe Epilepsy: Its Association with Psychiatric Impairment and Appropriate Dental Management," Oral Surgery, Oral Medicine, Oral Pathology, Vol. 68, No. 3, 1989, pp. 288-292. doi:10.1016/0030-4220(89)90213-2

[11] G. V. Busschots and B. I. Milzman, "Dental Patients with Neurologic and Psychiatric Concerns,” Dental Clinics of North America, Vol. 43, 1999, pp. 471-483.

[12] S. Gallagher, S. Weiss, C. J. Oram, T. Humphries, K. E. Harman and S. Menascu, "Efficacy of Very High Dose Steroid Treatment in a Case of Landau-Kleffner Syndrome," Developmental Medicine \& Child Neurology, Vol. 48, No. 9, 2006, pp. 766-769. doi:10.1017/S0012162206001630

[13] A. L. Lux and J. P. Osborne, "The Influence of Etiology upon Ictal Semiology, Treatment Decisions and LongTerm Outcomes in Infantile Spasms and West Syndrome,” Epilepsy Research, Vol. 70, 2006, pp. 77-86. doi:10.1016/j.eplepsyres.2006.01.017

[14] T. J. O’Brien, R. E. Hogan and L. Sedal, “Tooth-Brushing Epilepsy: A Report of a Case with Structural and Functional Imaging and Electrophysiology Demonstrating a Right Frontal Focus," Epilepsia, Vol. 37, No. 7, 1996, pp. 694-697. doi:10.1111/j.1528-1157.1996.tb00636.x

[15] G. L. Holmes, S. Blair and E. Eisenberg, "Tooth-Brushing-Induced Epilepsy,” Epilepsia, Vol. 23, No. 6, 1982, pp. 657-661. doi:10.1111/j.1528-1157.1982.tb05081.x

[16] M. Koutroumanidis, R. Pearce, D. R. Sadoh and P. Panayiotopoulos, "Tooth Brushinginduced Seizures: A Case Report,” Epilepsia, Vol. 42, No. 5, 2001, pp. 686688. doi:10.1046/j.1528-1157.2001.32700.x

[17] L. Pick and J. Bauer, "Dentistry and Epilepsy,” Nervenarzt, Vol. 72, No. 1, 2001, pp. 946-949. doi:10.1007/s001150170008

[18] B. T. Kennedy and J. S. Haller, "Treatment of the Epileptic Patient in the Dental Office," New York State Dental Journal, Vol. 64, 1998, pp. 26-31.

[19] K. N. Ciarroca, M. S. Greenberg and A. Garfunkel, "Neuromuscular Disease,” In: M. S. Greenberg, M. Glick, Eds., Burket's Oral Medicine Diagnosis and Treatment, 10th Edition, BC Decker Inc., Lewiston, 2003, p. 592.

[20] E. N. Arnautova and T. N. Nesmeianova, "A Proposed International Classification of Epileptic Seizures,” Epi- 
lepsia, Vol. 5, No. 4, 1964, pp. 297. doi:10.1111/j.1528-1157.1964.tb03337.x

[21] K. Karolyhazy, E. Kovacs, P. Kivovics, P. Fejerdy and Z. Aranyi, "Dental Status and Oral Health of Patients with Epilepsy: An Epidemiologic Study,” Epilepsia, Vol. 44, No. 8, 2003, pp. 1103-1108. doi:10.1046/j.1528-1157.2003.04003.x

[22] M. P. Majola, M. L. McFadyen, C. Connolly, Y. P. Nair, M. Govender and M. H. Laher, "Factors Influencing Phenytoin-Induced Gingival Enlargement,” Journal of Clinical Periodontology, Vol. 27, No. 7, 2000, pp. 506512. doi:10.1034/j.1600-051x.2000.027007506.x

[23] I. Marakoglu, U. K. Gursoy, H. Cakmak and K. Marakoglu, "Phenytoin-Induced Gingival Overgrowth in UnCooperated Epilepsy Patients,” Yonsei Medical Journal, Vol. 45, No. 2, 2004, pp. 337-340.

[24] J. French, "The Long-Term Therapeutic Management of Epilepsy,” Annals of Internal Medicine, Vol. 120, No. 5, 1994, pp. 411-422.

[25] M. J. Brodie, "Diagnosing and Predicting Refractory Epilepsy,” Acta Neurologica Scandinavica, Vol. 112, Suppl. 181, 2005, pp. 36-39. doi:10.1111/j.1600-0404.2005.00507.x

[26] R. H. Mattson, "Medical Management of Epilepsy in Adults,” Neurology, Vol. 51, No. ,5 1998, pp. 15-20.

[27] F. Perlik, M. Kolinova, J. Zvarova and V. Patzelova, "Phenytoin as a Risk Factor in Gingival Hyperplasia," Therapeutic Drug Monitoring, Vol. 17, No. 5, 1995, pp. 445-448. doi:10.1097/00007691-199510000-00002

[28] K. Karolyhazy, P. Kivovics, P. Fejerdy and Z. Aranyi, "Prosthodontic Status and Recommended Care of Patients with Epilepsy,” Journal of Prosthetic Dentistry, Vol. 93,
No. 2, 2005, pp. 177-182. doi:10.1016/j.prosdent.2004.11.008

[29] S. F. Malamed, "Handbook of Local Anesthesia," 4th Edition, Mosby, St Louis, 1997, p. 121.

[30] D. Buck, G. A. Baker, A. Jacoby, D. F. Smith and D. W. Chadwick, "Patients' Experiences of Injury as a Result of Epilepsy,” Epilepsia, Vol. 38, No. 4, 1997, pp. 439-444. doi:10.1111/j.1528-1157.1997.tb01733.x

[31] M. T. Hosey and R. R. Welbury, "Medical Disability,” In: R. R. Welbury, M. S. Duggal and M. T. Hosey, Eds., Pediatric Dentistry, 3rd Edition, Oxford University Press, Oxford, 2005, pp. 385-410.

[32] C. E. Dent, A. Richens, D. J. Rowe and T. C. Stamp, "Osteomalacia with Long-Term Anticonvulsant Therapy in Epilepsy,” British Medical Journal, Vol. 4, 1970, pp. 69-72. doi:10.1136/bmj.4.5727.69

[33] K. B. Desai, W. J. Ribbans and G. J. Taylor, "Incidence of Five Common Fracture Types in an Institutional Epileptic Population,” Injury, Vol. 27, 1996, pp. 97-100. doi:10.1016/0020-1383(95)00189-1

[34] J. A. Parry and F. A. Khan, "Provision of Dental Care for Medically Compromised Children in the UK by General Dental Practitioners," International Journal of Paediatric Dentistry, Vol. 10, No. 4, 2000, pp. 322-327. doi:10.1046/j.1365-263x.2000.00207.x

[35] B. J. Abdullah, L. K. Teong, J. Mahadevan and A. “Jalaludin, Dental Prosthesis Ingested and Impacted in the Esophagus and Orolaryngopharynx," Journal of Otolaryngology, Vol. 27, 1998, pp. 190-194. 